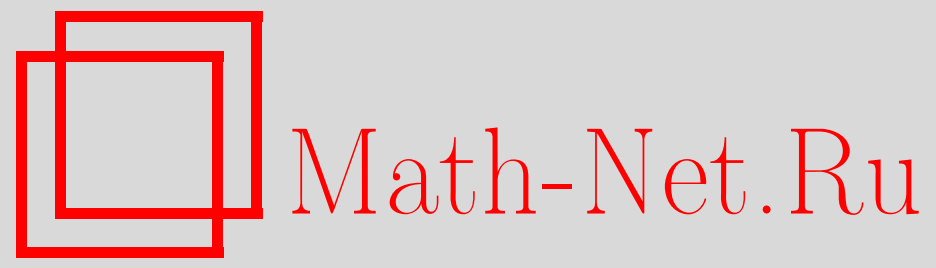

Обцероссийский математический портал 
С. В. Конягин, Аддитивная проблема с дробными степенями, Матем. заметки, 2003, том 73, выпуск 4, 633-636

DOI: https://doi.org/10.4213/mzm626

Использование Общероссийского математического портала Math-Net.Ru подразумевает, что вы прочитали и согласны с пользовательским соглашением http://www. mathnet.ru/rus/agreement 
Параметры загрузки:

IP : 54.209 .52 .79

26 апреля 2023 г., 16:35:31

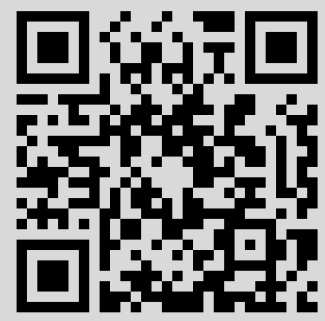




\section{АДДИТИВНАЯ ПРОБЛЕМА С ДРОБНЫМИ СТЕПЕНЯМИ}

\section{С. В. Конягин}

Для $u \in \mathbb{R}$ через $[u]$ и $\|u\|$ будем обозначать соответственно наибольшее целое число, не превосходящее $u$, и расстояние от $u$ до ближайшего целого числа. Ж.-М. Дезуе [1] доказал, что для любого $\alpha \in(1,4 / 3)$ всякое достаточно большое натуральное число $x$ представимо в виде $x=\left[U^{\alpha}\right]+\left[V^{\alpha}\right]$ с цельпи неотрицательньми $U$ и $V$. С.А. Гриценко [2] расширил область значений $\alpha$, обладающих этим свойством, до $\alpha \in(1,55 / 41)$. М.З. Гараев и Ка-Лам Куэ [3] показали, что для любых $\alpha \in(1,3 / 2]$ и $N \geqslant 2$ имеется $\gg N$ натуральных чисел $x \leqslant N$, представимых в виде $x=\left[U^{\alpha}\right]+\left[V^{\alpha}\right]$ с натуральными $U$ и $V$.

Целью настоящей работы является доказательство следующего результата.

Теорема. Для любых $\alpha \in(1,3 / 2)$ всякое достаточно большое натуральное число $x$ представимо в виде $x=\left[U^{\alpha}\right]+\left[V^{\alpha}\right]$ с натуральными $U$ u $V$. 
ДоКАЗАТЕльство. Возьмем $\beta=(3 / 2-\alpha) / 2$. Для натуральных чисел $k$ и $n$ с $n / 2 \leqslant k \leqslant n \leqslant x^{\beta}$ определим положительные числа $u_{0}=u_{0}(n, k)$ и $v_{0}=v_{0}(n, k)$ так, чтобы

$$
u_{0}^{\alpha}+v_{0}^{\alpha}=x \quad \text { и } \quad n u_{0}^{\alpha-1}=k v_{0}^{\alpha-1} .
$$

Таким образом,

$$
u_{0}=\frac{k^{1 /(\alpha-1)}}{L} x^{1 / \alpha}, \quad v_{0}=\frac{n^{1 /(\alpha-1)}}{L} x^{1 / \alpha}, \quad L=\left(k^{\alpha /(\alpha-1)}+n^{\alpha /(\alpha-1)}\right)^{1 / \alpha} .
$$

Заметим, что

$$
u_{0} \asymp v_{0} \asymp x^{1 / \alpha} .
$$

Обозначим $u=\left[u_{0}\right], v=\left[v_{0}\right]$. В силу (3) мы имеем

$$
0 \leqslant u_{0}^{\alpha}-u^{\alpha} \ll u_{0}^{\alpha-1} \ll x^{(\alpha-1) / \alpha}, \quad 0 \leqslant v_{0}^{\alpha}-v^{\alpha} \ll x^{(\alpha-1) / \alpha} .
$$

Следовательно,

$$
0 \leqslant x-u^{\alpha}-v^{\alpha} \ll x^{(\alpha-1) / \alpha} .
$$

Наша цель - показать, что для подходящих $n, k$ и натуральных чисел $w, U=u-w n, V=v+w k$ выполняется равенство

$$
x=\left[U^{\alpha}\right]+\left[V^{\alpha}\right] .
$$

Рассмотрим функцию

$$
f(w)=(u-w n)^{\alpha}+(v+w k)^{\alpha}, \quad 0 \leqslant w \leqslant \frac{u}{n} .
$$

Учитывая (3), имеем

$$
\begin{aligned}
(u-w n)^{\alpha} & =u^{\alpha}-\alpha u^{\alpha-1} w n+\frac{\alpha(\alpha-1)}{2} u^{\alpha-2}(w n)^{2}+O\left(u^{\alpha-3}(w n)^{3}\right) \\
& =u^{\alpha}-\alpha u_{0}^{\alpha-1} w n+O\left(u_{0}^{\alpha-2} w n\right)+\frac{\alpha(\alpha-1)}{2} u^{\alpha-2}(w n)^{2}+O\left(u^{\alpha-3}(w n)^{3}\right) \\
& =u^{\alpha}-\alpha u_{0}^{\alpha-1} w n+\frac{\alpha(\alpha-1)}{2} u^{\alpha-2}(w n)^{2}+O\left(x^{(\alpha-2) / \alpha} w n\right)+O\left(x^{(\alpha-3) / \alpha}(w n)^{3}\right) .
\end{aligned}
$$

Аналогично,

$$
(v+w k)^{\alpha}=v^{\alpha}+\alpha v_{0}^{\alpha-1} w k+\frac{\alpha(\alpha-1)}{2} v^{\alpha-2}(w k)^{2}+O\left(x^{(\alpha-2) / \alpha} w n\right)+O\left(x^{(\alpha-3) / \alpha}(w n)^{3}\right) .
$$

Здесь мы использовали, что $k \asymp n$. Суммируя (6) и (7) и пользуясь (1), получаем

$$
f(w)=u^{\alpha}+v^{\alpha}+\frac{\alpha(\alpha-1)}{2} w^{2}\left(u^{\alpha-2} n^{2}+v^{\alpha-2} k^{2}\right)+O\left(x^{(\alpha-2) / \alpha} w n\right)+O\left(x^{(\alpha-3) / \alpha}(w n)^{3}\right) .
$$

Возьмем $w n=A x^{1 /(2 \alpha)}$ с большим $A$. Из (8) следует, что

$$
f(w) \geqslant u^{\alpha}+v^{\alpha}+\frac{\alpha(\alpha-1)}{2} A^{2} u^{\alpha-2} x^{1 / \alpha}+O\left(x^{(\alpha-3 / 2) / \alpha} A^{3}\right) .
$$

В силу (3) существует такая постоянная $c=c(\alpha)>0$, что

$$
\frac{\alpha(\alpha-1)}{2} u^{\alpha-2} \geqslant c x^{(\alpha-2) / \alpha}
$$

Таким образом, при $x \rightarrow \infty$

$$
f(w) \geqslant u^{\alpha}+v^{\alpha}+c A^{2} x^{(\alpha-1) / \alpha}+O\left(x^{(\alpha-3 / 2) / \alpha} A^{3}\right)=u^{\alpha}+v^{\alpha}+\left(c A^{2}+o(1)\right) x^{(\alpha-1) / \alpha} .
$$

Из (4) вытекает, что если мы зафиксируем большое $A$, то для достаточно большого $x$ справедливо неравенство $f(w)>x+1$ и, кроме того, $f(0)<x+1$. Значит, найдется такое число $w_{0}>0$, что

$$
\left(u-w_{0} n\right)^{\alpha}+\left(v+w_{0} k\right)^{\alpha}=f\left(w_{0}\right)=x+1 \quad \text { и } \quad w_{0} n \ll x^{1 /(2 \alpha)} .
$$

Далее нам потребуется следующее неравенство. 
Лемма 1. Пусть $z_{0}>0, z_{1} \geqslant z_{0} / 2, z_{2} \geqslant z_{0} / 2$. Тогда

$$
\left|z_{1}^{\alpha}-z_{2}^{\alpha}-\alpha\left(z_{1}-z_{2}\right) z_{0}^{\alpha-1}\right| \ll\left|z_{1}-z_{2}\right| \max _{z \in\left[z_{1}, z_{2}\right]}\left|z-z_{0}\right| z_{0}^{\alpha-2}
$$

ДокАЗАТЕльство. Мы имеем

$$
z_{1}^{\alpha}-z_{2}^{\alpha}=\int_{z_{2}}^{z_{1}} \alpha z^{\alpha-1} d z .
$$

Следовательно,

$$
\begin{aligned}
\left|z_{1}^{\alpha}-z_{2}^{\alpha}-\alpha\left(z_{1}-z_{2}\right) z_{0}^{\alpha-1}\right| & \leqslant \int_{z_{2}}^{z_{1}}\left|\alpha z^{\alpha-1}-\alpha z_{0}^{\alpha-1}\right||d z| \\
& \leqslant\left|z_{1}-z_{2}\right| \max _{z \in\left[z_{1}, z_{2}\right]} \alpha\left|z^{\alpha-1}-z_{0}^{\alpha-1}\right| .
\end{aligned}
$$

Заметим, что поскольку $0<\alpha-1<1$, для $z \in\left[z_{1}, z_{2}\right]$ имеет место неравенство

$$
\left|z^{\alpha-1}-z_{0}^{\alpha-1}\right| \leqslant(\alpha-1)\left|z-z_{0}\right|\left(\min \left(z, z_{0}\right)\right)^{\alpha-2} \ll\left|z-z_{0}\right| z_{0}^{\alpha-2}
$$

и лемма доказана.

Обозначим $w_{1}=\left[w_{0}\right], w_{2}=\left[w_{0}\right]+1$. На основании леммы 1 мы можем написать несколько полезных неравенств.

Лемма 2. Существует такое $\varepsilon=\varepsilon(\alpha, x)$, что

$$
\varepsilon \ll n x^{(\alpha-3 / 2) / \alpha}
$$

и справедливы следующие неравенства:

$$
\begin{array}{cl}
\left|\left(u-w_{j} n\right)^{\alpha}-\left(u-w_{0} n\right)^{\alpha}-\alpha\left(w_{0}-w_{j}\right) n u_{0}^{\alpha-1}\right| \leqslant \varepsilon, & j=1,2, \\
\left|\left(v+w_{j} k\right)^{\alpha}-\left(v+w_{0} k\right)^{\alpha}-\alpha\left(w_{j}-w_{0}\right) k v_{0}^{\alpha-1}\right| \leqslant \varepsilon, & j=1,2 .
\end{array}
$$

Доказательство непосредственно следует из леммы 1 и (9). Заметим, что ввиду выбора $n$

$$
\varepsilon \ll x^{\gamma}, \quad \gamma=\frac{(\alpha-3 / 2)(2-\alpha)}{2 \alpha}<0 .
$$

Следуюшая лемма является решающей для доказательства теоремы.

ЛЕмма 3. Если $\left\|\alpha n u_{0}^{\alpha-1}\right\|>6 \varepsilon, m o\left[\left(u-w_{1} n\right)^{\alpha}\right]+\left[\left(v+w_{1} k\right)^{\alpha}\right]=x u \Omega u\left[\left(u-w_{2} n\right)^{\alpha}\right]+$ $\left[\left(v+w_{2} k\right)^{\alpha}\right]=x$.

ДоКАЗАТЕЛЬСтво. Суммируя (10) и (11) и учитьвая (1) и (9), получаем

$$
\left|\left(u-w_{j} n\right)^{\alpha}+\left(v+w_{j} k\right)^{\alpha}-x-1\right| \leqslant 2 \varepsilon, \quad j=1,2 .
$$

Рассмотрим случай, когда для некоторого $j=1,2$ справедливо неравенство $\left\|\left(u-w_{j} n\right)^{\alpha}\right\|>2 \varepsilon$. Тогда

$$
2 \varepsilon<\left\{\left(u-w_{j} n\right)^{\alpha}\right\}+\left\{\left(v+w_{j} k\right)^{\alpha}\right\}<2-2 \varepsilon .
$$

Значит, в силу (13)

$$
x-1<\left[\left(u-w_{j} n\right)^{\alpha}\right]+\left[\left(v+w_{j} k\right)^{\alpha}\right]<x+1,
$$

или $\left[\left(u-w_{j} n\right)^{\alpha}\right]+\left[\left(v+w_{j} k\right)^{\alpha}\right]=x$. Таким образом, заключение леммы справедливо, если только одновременно не выполнены неравенства

$$
\left\|\left(u-w_{1} n\right)^{\alpha}\right\| \leqslant 2 \varepsilon, \quad\left\|\left(u-w_{2} n\right)^{\alpha}\right\| \leqslant 2 \varepsilon .
$$


Достаточно изучить случай, когда имеет место (14) и, следовательно,

С другой стороны, в силу (10)

$$
\left\|\left(u-w_{1} n\right)^{\alpha}-\left(u-w_{2} n\right)^{\alpha}\right\| \leqslant 4 \varepsilon .
$$

$$
\left|\left(u-w_{1} n\right)^{\alpha}-\left(u-w_{2} n\right)^{\alpha}-\alpha n u_{0}^{\alpha-1}\right| \leqslant 2 \varepsilon .
$$

Таким образом, $\left\|\alpha n u_{0}^{\alpha-1}\right\| \leqslant 6 \varepsilon$, и лемма доказана.

Возьмем $\boldsymbol{n}=\left[x^{\beta}\right]$. Для доказательства теоремы достаточно убедиться в существовании такого целого $k \in[n / 2, n]$, что

где $\varepsilon$ удовлетворяет (12). Пусть

$$
\left\|\alpha n u_{0}^{\alpha-1}\right\|>6 \varepsilon
$$

$$
g(u)=\frac{\alpha u}{\left(1+u^{\alpha /(\alpha-1)}\right)^{(\alpha-1) / \alpha}} .
$$

В силу (2) неравенство (16) может быть переписано в виде

$$
\left\|n x^{(\alpha-1) / \alpha} g\left(\frac{k}{n}\right)\right\|>6 \varepsilon .
$$

Возьмем $l=[(\alpha-1) /(\alpha \beta)]+2$ и интервал $\left[u_{1}, u_{2}\right] \subset(1 / 2,1)$ такой, что $u_{2}>u_{1}$ и $g^{(j)}(u) \neq 0$ при $u \in\left[u_{1}, u_{2}\right], j=1, \ldots, l$. Тогда для тригонометрической суммы

$$
S=\sum_{u_{1} n \leqslant k \leqslant u_{2} n} e\left(n x^{(\alpha-1) / \alpha} g\left(\frac{k}{n}\right)\right),
$$

где $e(u)=e^{2 \pi i u}$, имеет место оценка [4, теорема 5 главы 1$]$

$$
|S| \ll n\left(n^{-l} F\right)^{1 /\left(2^{l}-2\right)}+n \times n^{-1 / 2^{l-1}}\left(n^{-l} F\right)^{-1 /\left(2^{l+1}-2\right)},
$$

где $F=n x^{(\alpha-1) / \alpha}$. Ввиду выбора $l$ мы имеем $n^{-1} \ll n^{-l} F=o(1)$. Отсюда при $n \rightarrow \infty$

$$
\begin{gathered}
\left(n^{-l} F\right)^{1 /\left(2^{l}-2\right)}=o(1), \\
n^{-1 / 2^{l-1}}\left(n^{-l} F\right)^{-1 /\left(2^{l+1}-2\right)} \ll n^{-1 / 2^{l-1}}\left(n^{-l} F\right)^{-1 / 2^{l}} \ll n^{-1 / 2^{l}}=o(1)
\end{gathered}
$$

и, вследствие (18), $S=o(n)$ при $n \rightarrow \infty$. Значит, для большого $n$ найдется такое $k \in\left[u_{1} n, u_{2} n\right]$, что

$$
\left\|n x^{(\alpha-1) / \alpha} g\left(\frac{k}{n}\right)\right\|>0.2>6 \varepsilon .
$$

Действительно, если мы допустим противное, то

$$
\Re S \geqslant \sum_{u_{1} n \leqslant k \leqslant u_{2} n} \cos (0.4 \pi) \gg n,
$$

но последнее неравенство противоречит установленной оценке $S=o(n)$. Итак, неравенство (17) выполнено для некоторого $k$. Тем самым теорема доказана.

Автор выражает глубокую благодарность математическому институту Макса Планка в Бонне, где была подготовлена настоящая статья, за гостеприимство и прекрасные условия для работы. Автор благодарен также профессору Д. Толеву за интерес к работе и ряд полезных советов и замечаний.

\section{СПИСОК ЦИТИРОВАННОЙ ЛИТЕРАТУРЫ}

1. Deshouillers J.-M. // Acta Arith. 1973-1974. V. 25. P. 393-403. 2. Гриценко С. А. // Изв. РАН. Сер. матем. 1993. Т. 41. № 3. С. 447-464. 3. Garaev M. Z., Ka-Lam Kueh. // J. Anal. Appl. 2001. V. 20. Р. 999-1006. 4. Карацуба А. А. Основы аналитической теории чисел. M.: Наука, 1983. 